\title{
Evaluation of Waterflood Development Effect by Using Recovery Degree of Recoverable Reserves and Cumulative Water Storage Rate
}

\author{
Lijun Huang \\ The 3rd Oil Production Factory, Daqing Oil Field Company Ltd, Daqing, China
}

\begin{abstract}
For water flooded oilfields, the development effect can be given by analyzing the relationship between the recoverable reserves recovery degree and the cumulative water storage rate and water content. Application of recoverable reserves as the basis for evaluating development effects is more comparable than the extent of geological reserves; The cumulative water storage rate is suitable for evaluating the evaluation unit as a whole since its development. Correctly deriving the relationship between the extent of recoverable reserves recovery and the cumulative water storage and water content are the basic conditions for its application.
\end{abstract}

\section{Selection basis of water drive evaluation indexes}

In the past, the comparison of the relationship between water content and the extent of geological reserve extraction was used as the basis for evaluating development effects, but it is not comparable because of different underground conditions in the reservoir. The degree of recovery with recoverable reserves is comparable. Because regardless of the amount of geological reserves, the recoverable reserves under the current state are certain, so they are comparable Cumulative water storage rate is the ratio of the difference between cumulative water injection and cumulative water production to cumulative water injection. The cumulative water storage rate is suitable for evaluating the evaluation unit as a whole since its development. In general, if the oil field has a high cumulative water storage rate, the degree of recovery will be high, and the water content will be low, and the injection water will be highly efficient, and the development effect is good; on the other hand, if the oil field has a low cumulative water storage rate, it has a low recovery rate and high water content injected water has low utilization efficiency and poor development effect (Figure 1).

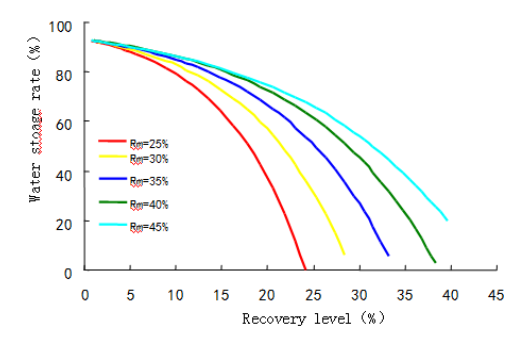

Figure 1 theoretical relation curve between cumulative water storage rate and recovery degree

\section{Determination of evaluation standard of water drive theory}

\subsection{Derivation of expression of recovery degree and cumulative water storage rate of recoverable reserves}

Water drive curve is the main method to study the law of water cut change in water drive oilfield. The relationship between recoverable reserves recovery degree, cumulative water storage rate and comprehensive water cut derived from water drive curve can reflect the characteristics of water drive development in Oilfield. In the following, taking the C-type water drive characteristic curve as an example, the expressions of recoverable reserves recovery degree and cumulative water storage rate are deduced. The relationship between cumulative oil production and cumulative liquid production is

$$
\frac{L_{\mathrm{P}}}{N_{\mathrm{P}}}=a+b L_{\mathrm{P}}
$$


In the formula, $\mathrm{a}$ and $\mathrm{B}$ are characteristic curve coefficients of C-type water drive; NP and LP are cumulative oil production and liquid production $\left(10^{4} \mathrm{t}\right)$.

By transforming formula (1) and deriving the time, we get

$$
N_{P}=\frac{1}{b}\left[1-\sqrt{a\left(1-f_{W}\right)}\right]
$$

Where $f_{\mathrm{w}}$ is water content $(\%)$.

When the ultimate water cut of oil field is $98 \%$, the formula of recoverable reserves is obtained.

$$
N_{R}=\frac{1}{b}\left[1-\frac{\sqrt{2 a}}{10}\right]
$$

Where $N_{\mathrm{R}}$ is recoverable reserves $\left(10^{4} \mathrm{t}\right)$.

The relationship between recovery degree of recoverable reserves and water cut can be obtained by dividing (2) (3)

$$
R_{N R}=\frac{1-\sqrt{a\left(1-f_{W}\right)}}{1-\sqrt{2 a} / 10}
$$

where $R_{N R}$ is the recovery degree of recoverable reserves.

Formula (4) is the theoretical evaluation standard of recovery degree and water cut of recoverable reserves.

Water storage rate is an important index to evaluate the development effect of waterflooding oilfield.

The cumulative water storage rate is defined as

$$
E_{S}=\frac{W_{i}-W_{p}}{W_{i}}=1-\frac{W_{P}}{W_{i}}
$$

Cumulative bet ratio is defined as

$$
Z_{S}=\frac{W_{i}}{L_{P}}
$$

Substituting formula (6) into formula (5)

$$
E_{S}=1-\frac{1-\frac{N_{P}}{L_{P}}}{Z_{S}}
$$

Substituting formula (1) into formula (7)

$$
E_{S}=1-\frac{a+b N_{P}-1}{a Z_{S}}
$$

Substituting formula (2) into formula (8)

$$
E_{S}=1-\frac{a-\sqrt{a\left(1-f_{W}\right)}}{a Z_{S}}
$$

In the formula, $E_{\mathrm{S}}$ is cumulative water storage rate $(\%) ; Z_{\mathrm{S}}$ is cumulative injection production ratio.

Equation (9) is the relationship between accumulated water storage rate and water content. The cumulative water storage rate is not only related to water cut, but also to the coefficient of C-type water drive characteristic curve. When other conditions are certain, the cumulative water storage rate increases with the increase of cumulative injection production ratio.

\subsection{Selection of cumulative water storage rate for water drive characteristic curve}

Based on the mathematical derivation of the other five water drive characteristic curves recommended in the oil and natural gas standard, the relationship between the cumulative water storage rate and the water content is obtained. According to the expressions of six kinds of cumulative water storage rate, the theoretical relation chart is fitted. It can be seen that the C-type water drive characteristic curve is suitable for the prediction and evaluation of the relationship between cumulative water storage rate and water cut. The water drive characteristic curves of type A and type B can't reflect the correct relationship between accumulated water storage and water cut in low water cut stage, while those of type D, Zhang Jinqing and Yu Qitai can't correctly represent the relationship between them in medium high water cut stage.

\section{Development effect evaluation of Development Zone G}

\subsection{Evaluation on recovery degree of recoverable reserves}

The values of a, $b$ and $R_{\mathrm{NR}}$ of 6 blocks in the development zone are obtained by dynamic data fitting, as shown in Table 1.

Table 1 fitting a, B and R2 (fitting coefficient) parameter results of each block

\begin{tabular}{|c|c|c|c|c|c|c|c|}
\hline Block & $\mathrm{a}$ & $\mathrm{b}$ & $\mathrm{R} 2$ & $\mathrm{fW}$ & $\begin{array}{c}\text { Theory } \\
\text { RNR }\end{array}$ & $\begin{array}{c}\text { Actual } \\
\text { RNR }\end{array}$ & $\mathrm{R}$ \\
\hline (A) & 1.6406334 & 0.0002706 & 0.99977 & $95.25 \%$ & $88.03 \%$ & $91.00 \%$ & $40.56 \%$ \\
\hline (B) & 1.6243483 & 0.0001738 & 0.99981 & $95.63 \%$ & $89.49 \%$ & $90.74 \%$ & $41.33 \%$ \\
\hline (C) & 1.4258130 & 0.0001988 & 0.99959 & $92.26 \%$ & $80.35 \%$ & $92.57 \%$ & $34.12 \%$ \\
\hline (D) & 1.3982903 & 0.0002282 & 0.99964 & $92.64 \%$ & $81.56 \%$ & $92.54 \%$ & $41.59 \%$ \\
\hline (E) & 2.2803966 & 0.0003755 & 0.99972 & $95.51 \%$ & $91.28 \%$ & $91.68 \%$ & $36.33 \%$ \\
\hline (F) & 2.0071406 & 0.0001955 & 0.99988 & $95.91 \%$ & $91.07 \%$ & $91.96 \%$ & $37.39 \%$ \\
\hline
\end{tabular}

There is little difference in the recovery degree of the current recoverable reserves in six blocks. On the whole, by comparing the theoretical RNR with the actual value of the same block, under the same water cut condition, the recovery degree of the actual recoverable reserves of each block is greater than that of the theoretical recoverable reserves, indicating that the development effect of the six blocks is better. Block $\mathrm{C}$ and block $\mathrm{d}$ have the best development effect. The actual RNR value exceeds the theoretical value by $10 \%$, and the recovery degree of geological reserves in Block D is the highest in the whole area. Although the actual RNR value of block 
a and block B is more than $90 \%$, the actual RNR value is only 1-2 percentage points higher than the theoretical value, so the development effect is relatively general. Although the geological recovery degree $r$ of block $f$ is not much different from that of Block $\mathrm{C}$, the development effect of block $\mathrm{f}$ is worse than that of Block C because its actual $R_{N R}$ is similar to the theoretical value. The actual $R_{N R}$ of Block E is the closest to the theoretical value and the development effect is the worst. Comparing the recovery degree of different blocks with similar water content, it can be found that the recovery degree of recoverable reserves in Block $\mathrm{C}$ and block $\mathrm{D}$ is 3-4 percentage points higher than that in block $\mathrm{F}$ and block $\mathrm{E}$. In conclusion, block $\mathrm{C}$ and block $\mathrm{d}$ have the best development effect, and Block $\mathrm{E}$ has the worst development effect. In general, the development effect of pure oil area under the similar water content is better than that of transition zone.

\subsection{Evaluation with accumulated water storage rate}

The trend of change of cumulative water storage rate is restricted by the development system, if the actual water storage rate change trend is basically consistent with the theoretical curve, the water storage rate change will be also relatively stable. That means that the water drive effect is better, and the development system selection is reasonable, and it is conducive to the rational exploitation of oil fields, and the development system should continue to be maintained. It reflects that the water injection condition of the oil field is not stable, which leads to the poor utilization rate of the injection water and the poor effect of water drive. From the change curve of the theoretical water storage rate and the actual water storage rate of each block, it can be seen that the actual water storage rate is basically consistent with the change trend of the theoretical curve, which shows that the water drive effect of 6 blocks is good and the water injection utilization rate is high. In 1980-1990, the cumulative water storage rate of each block continued to decrease, the development effect gradually deteriorated, the analysis was due to the "seven-five-year" period of comprehensive pumping, resulting in a decrease in the cumulative water storage rate of the formation;

Secondary encryption wells were put into operation, and there are about $40 \%$ of the thickness of the new and old wells at the same time. They played a role in the new and old wells between the water injection structural adjustment. The "steady oil control water" project has been effectively controlled, and the cumulative water storage rate is slowly decreased. During the period 1980-1990, the cumulative water storage rate of Block B decreased faster than that of other blocks in the pure oil area, and the development effect was poor, the analysis was due to the low cumulative injection ratio due to the imbalance of injection. In 1990, the cumulative injection ratio of Block B was 1.14, while the average cumulative injection ratio of the other three blocks in the pure oil area was 1.52. Block A cumulative water storage rate and $\mathrm{C}$ block and $\mathrm{D}$ block trend is similar, but the theoretical recoverable reserves extraction degree and their difference is quite large, the analysis is that the cumulative injection is relatively high (in the last 10 years has been greater than 1.4) resulting in high water storage rate calculation results, it need to take measures such as sealing and filling holes to achieve efficient water injection. Compared with pure oil area, the initial theoretical accumulation of water storage rate is low, and the actual water storage rate is declining fast, the analysis is mainly due to the intrusion of side water, bottom water, this part of the invasive water for the reservoir is equivalent to the injection of water, but not included in the cumulative injection of water, but increased the yield of water, resulting in low cumulative injection ratio. According to the definition of theoretical cumulative water storage rate, the comprehensive water content is high, the cumulative injection yield is low, and the theoretical cumulative water storage rate is certainly low. And according to the definition of the actual cumulative water storage rate, the cumulative water storage rate must be low if the water production increases.

\section{Conclusion}

4.1 Correctly deriving the relationship between the extent of recoverable reserves recovery and the cumulative water storage rate and water content are the basic conditions for evaluating the effect of water flooding.

4.2 Type $\mathrm{C}$ water flooding characteristic curve is suitable for prediction and evaluation of cumulative water storage rate.

\section{References}

1. Zhang Rui. A method to evaluate and predict the effect of oilfield water injection [J]. Petroleum exploration and development, 1985, 12 (4): 36-52.

2. Xingjun, song Ziqi, Cheng Zhongping. Comprehensive evaluation method of water drive development effect of sandstone reservoir [J]. Petroleum exploration and development, 2003, 30 (2): 68-69. 